International Journal of Pure and Applied Mathematics

Volume 87 No. 2 2013, 195-204

ISSN: $1311-8080$ (printed version); ISSN: 1314-3395 (on-line version)

url: http://www.ijpam.eu

doi: http://dx.doi.org/10.12732/ijpam.v87i2.1

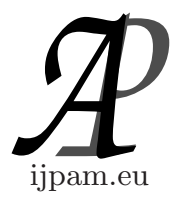

\title{
GENERAL ANALYSIS OF TWO PRODUCTS INVENTORY SYSTEM WITH SEASONAL PRODUCTION AND SALES
}

\author{
S. Parvathi ${ }^{1}$, R. Ramanarayanan ${ }^{2}$, S. Srinivasaraghavan ${ }^{3}$ \\ ${ }^{1}$ Department of Mathematics \\ VelTech MultiTech Dr. Rangarajan Dr. Sakuntala Engineering College \\ Avadi, Chennai, Tamil Nadu, INDIA \\ ${ }^{2,3}$ Department of Mathematics \\ VelTech Dr. RR and Dr. SR Technical University \\ Avadi, Chennai, Tamil Nadu, INDIA
}

\begin{abstract}
In this paper we discuss a two different products inventory system where the production has the impact of seasonal demand and sales. We consider the following model in which the production and sales of $k$ pairs of two different products are studied subject to seasonal requirements. The production of the two different products is done alternately. The production times of the products have general distribution and the seasonal duration is exponential. The double Laplace Transform of production and sale time and their means are obtained. Numerical examples are presented.
\end{abstract}

AMS Subject Classification: $30 \mathrm{C} 45,30 \mathrm{C} 80$

Key Words: inventory systems, two different products, seasonal sales

\section{Introduction}

Several researchers studied single commodity inventory systems of $(s, S)$ type. Arrow, Karlin and Scart [1] first analysed such inventory systems. Daniel and Ramanarayanan [2] discussed $(s, S)$ inventory system with random lead times and unit demand models, Bulk demand models were treated by Ramanarayanan

Received: August 9, 2012

(C) 2013 Academic Publications, Ltd.

$\S_{\text {Correspondence author }}$ url: www.acadpubl.eu 
and Jacob [8]. Murthy and Ramanarayanan [4, 5, 6, 7] considered several $(s, S)$ inventory systems. Kun-Shan Wu and Liang-yuh ouyang [3] studied $(Q, R, L)$ inventory model with defective items. Murthy and Ramanarayanan [8] treated the $(s, S)$ inventory system with defective supplies where the supply was accepted only when at least $S+1$ units were good. In real life inventory models different types of pairs of products are produced for sales by the companies. For example drug manufacturing companies produce antibiotic and vitamin tablets. Oil refining companies produce fuel oil like petrol and engine oil to reduce friction and auto mobile companies produce engine and body of vehicles. In textiles there is demand for shirting cloth and pant cloth, inner garments and outer garments and so on. In this paper we study inventory systems and their related inventory subject to seasonal demands. In section 1 the double Laplace Transform of production of $k$ pairs with seasonal threshold and sales is presented. The expected production time and expected sale time and their product moment are obtained. In section 2 Numerical examples are presented.

\section{Model}

\subsection{Assumptions: Production and Sales}

The following are the assumptions of the model

(i) The company produces two different products $A$ and $B$ and at a time only one type is produced. The production time $X$ of product $A$ is random variable with $\mathrm{Cdf} G_{X}(x)$ and the production time $Y$ of product $B$ has Cdf $G_{Y}(x)$. $A$ and $B$ are produced in successive manner. The production time $X+Y$ of a pair has $\operatorname{Cdf} G(x)$.

(ii) The demand season for the products starts in an exponential time with parameter c from the starting time zero.

(iii) Sale time starts when $k$ number of pairs of products are produced or when the demand season starts.

(iv) The products are sold in pairs and the selling time of a pair is random variable with $\operatorname{Cdf} R(y)$ and the Cdf of selling time of product $A$ is $R_{A}(y)$.

We note that the probability of $n$ number of pairs produced in $(o, t)=$ $G_{n}(t)-G_{n+1}(t)$ where $G_{n}(t)$ is the Cdf of $\sum_{i=1}^{n}\left(X_{i}+Y_{i}\right)$. When the $n^{t h}$ pair is 
produced at time $x<t$ during $t-x$ there are two possibilities. After the $n^{t h}$ pair production,

(i) The production for product $A$ is over but for $B$ is not over or

(ii) The production for product $A$ is also not over.

Their respective probabilities are given below. We note that the probability of $n$ number of pairs and one production of $A$ is over before $t$, but production for $B$ is not over before $t$ is

$$
\begin{aligned}
& P\left[\sum_{i=1}^{n}\left(X_{i}+Y_{i}\right)+X_{n+1}<t<\sum_{i=1}^{n+1}\left(X_{i}+Y_{i}\right)\right] \\
& \quad=\int_{0}^{t} g_{n}(x) \int_{0}^{t-x} g_{X}(u) \overline{G_{Y}}(t-x-u) d u d x
\end{aligned}
$$

$=P[(n+1)$ number of $A$ products and $n$ number of $B$ products are produced during $(0, t)]$, Here $\bar{G}(x)=1-G(x)$.

Here $g_{n}(x)$ is the pdf of $\sum_{i=1}^{n}\left(X_{i}+Y_{i}\right)$ are produced in $(0, t)$. Also the probability of $n$ number of pairs are produced in $(0, t)$ and the $(n+1)^{t h}$ production of $A$ is not completed before $t$ is

$$
=\int_{0}^{t} g_{n}(x) \overline{G_{X}}(t-x) d x .
$$

Since the selling time starts when $k$ pairs are produced or when the season starts, the time to start sales $T=\operatorname{Min}$ (Time to produce $k$ pairs, the time at which the season starts).

The pdf of $T$ is

$$
\begin{aligned}
f_{T}(t) & =g_{k}(t) e^{-c t}+c e^{-c t} \sum_{i=0}^{k-1} \int_{0}^{t} g_{i}(x) \int_{0}^{t-x} g_{X}(u) \overline{G_{Y}}(t-x-u) d u d x \\
& +c e^{-c t} \sum_{i=0}^{k-1} \int_{0}^{t} g_{i}(x) \overline{G_{X}}(t-x) d x .
\end{aligned}
$$

The first term of the right side of (3) is the part of the pdf that the time to produce $k$ number of pairs is $t$ and the season has not started upto time $t$. The second term is the part of the pdf that the season starts at time $t$, the time to produce $i$ number of $A$ and $B$ pairs is $x$, the $(i+1)^{t h} A$ product is produced 
at time $x+u$ and the production of the $(i+1)^{t h} B$ product is not over during $t-x-u$ for $0 \leq i \leq k-1$. The third term is the part of the pdf that the season starts at time $t$, the time to produce $i$ number of $A$ and $B$ pairs is $x$ and the $(i+1)^{t h} A$ product is not over during $t-x$.

This gives the joint pdf of time to start sales $T$ and total sales time of pairs $R$ as follows considering the sales time of the pairs.

$$
\begin{aligned}
f_{T, R}(t) & =g_{k}(x) e^{-c x} r_{k}(y) \\
+ & c e^{-c x} \sum_{i=0}^{k-1} \int_{0}^{x} g_{i}(u) \int_{0}^{x-u} g_{X}(v) \overline{G_{Y}}(x-u-v) d v d u \\
& \quad \times \int_{0}^{y} r_{i}(w) r_{A}(y-w) d w \\
+ & c e^{-c x} \sum_{i=0}^{k-1} \int_{0}^{x} g_{i}(x) \overline{G_{X}}(x-u) d u r_{i}(y)
\end{aligned}
$$

The double Laplace transform of the pdf is given by

$$
\begin{aligned}
f_{T, R}^{*}(\epsilon, \eta)= & \int_{0}^{\infty} \int_{0}^{\infty} e^{-\epsilon x} e^{-\eta y} f_{T, R}(x, y) d x d y \\
= & \int_{0}^{\infty} \int_{0}^{\infty} e^{-\epsilon x-\eta y} g_{k}(x) e^{-c x} r_{k} d x d y \\
& +\int_{0}^{\infty} \int_{0}^{\infty} e^{-\epsilon x-\eta y} c e^{-c x} \sum_{i=0}^{k-1} \int_{0}^{x} g_{i}(u) \\
& \int_{0}^{x-u} g_{X}(v) \overline{G_{Y}}(x-u-v) d v d u \int_{0}^{y} r_{i}(w) r_{A}(y-w) d w d x d y \\
& +\int_{0}^{\infty} \int_{0}^{\infty} e^{-\epsilon x-\eta y} c e^{-c x} \sum_{i=0}^{k-1} \int_{0}^{x} g_{i}(u) \overline{G_{X}}(x-u) d u r_{i}(y) d x d y
\end{aligned}
$$

We get

$$
\begin{aligned}
f_{T, R}^{*}(\epsilon, \eta) & =g^{* k}(\epsilon+c) r^{* k}(\eta) \\
& +c \sum_{i=0}^{k-1} g^{* i}(\epsilon+c) g_{X}^{*}(\epsilon+c) \overline{G_{Y}^{*}}(\epsilon+c) r^{* i}(\eta) r_{A}^{*}(\eta) \\
& +c \sum_{i=0}^{k-1} g^{* i}(\epsilon+c) \overline{G_{X}^{*}}(\epsilon+c) r^{*}(\eta)
\end{aligned}
$$




$$
\begin{aligned}
f_{T, R}^{*}(\epsilon, \eta)=g^{* k} & (\epsilon+c) r^{* k}(\eta)+c\left[\frac{1-\left(g^{*}(\epsilon+c) r^{*}(\eta)\right)^{k}}{1-g^{*}(\epsilon+c) r^{*}(\eta)}\right] \\
& \times\left[g_{X}^{*}(\epsilon+c) \overline{G_{Y}^{*}}(\epsilon+c) r_{A}^{*}(\eta)+\overline{G_{X}^{*}}(\epsilon+c)\right]
\end{aligned}
$$

The Laplace transform of $T$ is

$$
\begin{aligned}
f_{T, R}^{*}(\epsilon, 0)=g^{*} & (\epsilon+c)+c\left[\frac{1-g^{* k}(\epsilon+c)}{1-g^{*}(\epsilon+c)}\right] \\
& \times\left[g_{X}^{*}(\epsilon+c) \overline{G_{Y}^{*}}(\epsilon+c)+\overline{G_{X}^{*}}(\epsilon+c)\right]
\end{aligned}
$$

On differentiation of equation (6) we get, $\frac{\partial}{\partial \epsilon} f_{T, R}^{*}(0,0)=-E(T)$ and we obtain

$$
\begin{aligned}
E(T) & =-k g^{k-1}(c) g^{*}(c)+c\left[\frac{k g^{*-1}(c) g^{*}(c)}{1-g^{*}(c)}\right]\left[g_{X}^{*}(c) \overline{G_{Y}^{*}}(c)+\overline{G_{X}^{*}}(c)\right] \\
& -c \frac{1-g^{* k}(c)}{\left(1-g^{*}(c)\right)^{2}} g^{*}(c)\left[g_{X}^{*}(c) \overline{G_{Y}^{*}}(c)+\overline{G_{X}^{*}}(c)\right] \\
& +c \frac{1-g^{* k}(c)}{\left(1-g^{*}(c)\right)}\left[g_{X}^{*}(c) \overline{G_{Y}^{*}}(c)+g_{X}^{*}(c) \overline{G_{Y}^{*}}(c)+\overline{G_{X}^{*}}(c)\right]
\end{aligned}
$$

Similarly we note $\frac{\partial}{\partial \eta} f_{T, R}^{*}(0,0)=-E(R)$ and we obtain

$$
\begin{aligned}
E(R) & =g^{* k}(c) k E\left(R_{1}\right)-c k E\left(R_{1}\right) \frac{g^{* k}(c)}{1-g^{*}(c)}\left[g_{X}^{*}(c) \overline{G_{Y}^{*}}(c)+\overline{G_{X}^{*}}(c)\right] \\
& +c E\left(R_{1}\right) \frac{1-g^{* k}(c)}{\left(1-g^{*}(c)\right)^{2}} g^{*}(c)\left[g_{X}^{*}(c) \overline{G_{Y}^{*}}(c)+\overline{G_{X}^{*}}(c)\right] \\
& -c \frac{1-g^{* k}(c)}{1-g^{*}(c)}\left[g_{X}^{*}(c) \overline{G_{Y}^{*}}(c)\left(-E\left(R_{1}\right)\right)\right]
\end{aligned}
$$

In most of stochastic models the Cdfs are exponential. In all the above cases we have fixed $k$ as a constant and we have considered sales for the system when it completes the production of $k$ pairs. As a most general case we may treat $k$ as a random variable taking positive integral values such that $p(k=i)=p_{i}>0$, $i>0$ and $\sum_{i=1}^{n} p_{i}=1$.

We now consider the special case in which $X$ and $Y$ are exponential random variables with parameters $a$ and $b$ respectively which gives

$$
g_{X}^{*}(c)=\frac{a}{a+c} \quad g_{X}^{*}=\frac{-a}{(a+c)^{2}}
$$




$$
\begin{aligned}
\overline{G_{Y}^{*}}(c) & =\frac{1}{b+c} & \overline{G_{Y}^{*}}(c) & =\frac{-1}{(b+c)^{2}} \\
\overline{G_{X}^{*}}(c) & =\frac{1}{a+c} & \overline{G_{X}^{*}}(c) & =\frac{-1}{(a+c)^{2}}
\end{aligned}
$$

Using (7), (8) and (9) we find $E(T)$ and $E(R)$, when $X$ and $Y$ are exponentials with parameters $a$ and $b$ as follows.

$$
E(T)=\frac{1-\left(\frac{a b}{(a+c)(b+c)}\right)^{k}}{(a+c)(b+c)}\left(\frac{a b}{c}+a+b+c\right)
$$

and

$$
E(R)=\left[1-\left(\frac{a b}{(a+c)(b+c)}\right)^{k}\right] \frac{a\left(b E\left(R_{1}\right)+c E\left(R_{A}\right)\right)}{c(a+b+c)}
$$

\section{Numerical Example}

To illustrate the applications of the above result we give different values for $a, b, c$ and $k$ and we obtain $E(T), E(R)$ in the following table. For $E\left(R_{1}\right)=20$ and $E\left(R_{0}\right)=10$

\begin{tabular}{|c|c|c|c|c|c|}
\hline $\mathrm{a}$ & $\mathrm{b}$ & $\mathrm{C}$ & $\mathrm{k}$ & $\mathrm{E}(\mathrm{T})$ & $\mathrm{E}(\mathrm{R})$ \\
\hline 1 & 2 & 3 & 4 & 0.3333 & 3.8885 \\
\hline 1 & 2 & 3 & 5 & 0.33333 & 3.88885 \\
\hline 1 & 2 & 3 & 6 & 0.333333 & 3.888885 \\
\hline 1 & 2 & 3 & 7 & 0.3333333 & 3.88888885 \\
\hline 1 & 2 & 3 & 8 & 0.33333333 & 3.88888885 \\
\hline 1 & 2 & 3 & 9 & 0.333333333 & 3.8888888885 \\
\hline
\end{tabular}




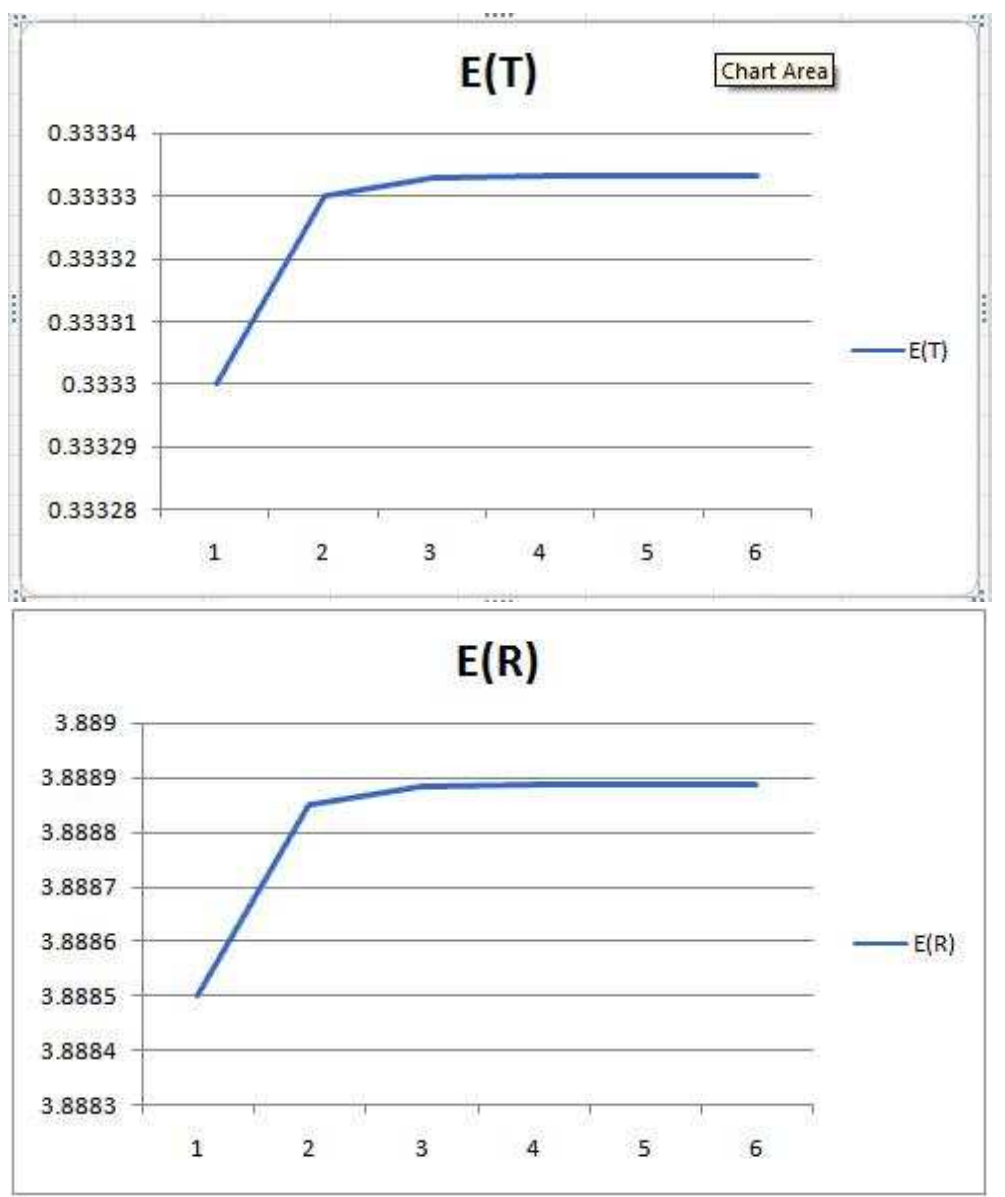

When $k$ is a random variable with $P(k=i)=p_{i} \geq 0, i \geq 1$ and $\sum_{i=1}^{\infty} p_{i}=1$ then let us consider $\phi(v)=\sum_{i=1}^{\infty} p_{i} r^{i}$. From equation (10) and (11) we get

$$
E(T)=\frac{1-\phi\left(\frac{a b}{(a+c)(b+c)}\right)}{(a+c)(b+c)}\left(\frac{a b}{c}+a+b+c\right)
$$

Similarly

$$
E(R)=\left[1-\phi\left(\frac{a b}{(a+c)(b+c)}\right)\right] \frac{a\left(b E\left(R_{1}\right)+c E\left(R_{A}\right)\right)}{c(a+b+c)}
$$


When $p_{i}=\frac{1}{2^{i}}$ for $i \geq 1$ we get $\phi(r)=\sum_{i=1}^{\infty} \frac{1}{2^{i}} r^{i}=\frac{r}{2-r}$ We obtain

$$
\begin{gathered}
E(T)=\frac{2 c(a+b+c)\left(\frac{a b}{c}+a+b+c\right)}{(a b+2 c(a+b+c))(a+c)(b+c)} \\
E(R)=\frac{2 c(a+b+c)}{(a b+2 c(a+b+c))} a\left(b E\left(R_{1}\right)+c E\left(R_{A}\right)\right)
\end{gathered}
$$

To illustrate the applications of the above result we give different values for $a, b, c$ and we obtain $E(T), E(R)$ in the following table.

\begin{tabular}{|c|c|c|c|c|}
\hline $\mathrm{a}$ & $\mathrm{b}$ & $\mathrm{C}$ & $\mathrm{E}(\mathrm{T})$ & $\mathrm{E}(\mathrm{R})$ \\
\hline 1 & 2 & 3 & 0.3157 & 6.6311 \\
\hline 1 & 2 & 4 & 0.2413 & 7.7240 \\
\hline 1 & 2 & 5 & 0.19512 & 8.7804 \\
\hline 1 & 2 & 6 & 0.1408 & 9.8181 \\
\hline 1 & 2 & 7 & 0.1235 & 10.845 \\
\hline
\end{tabular}

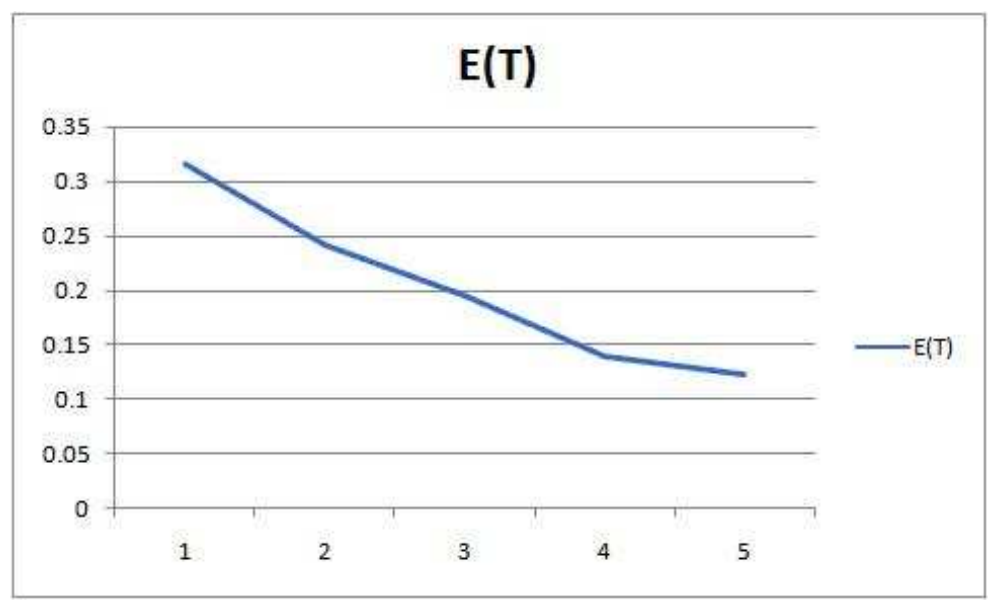




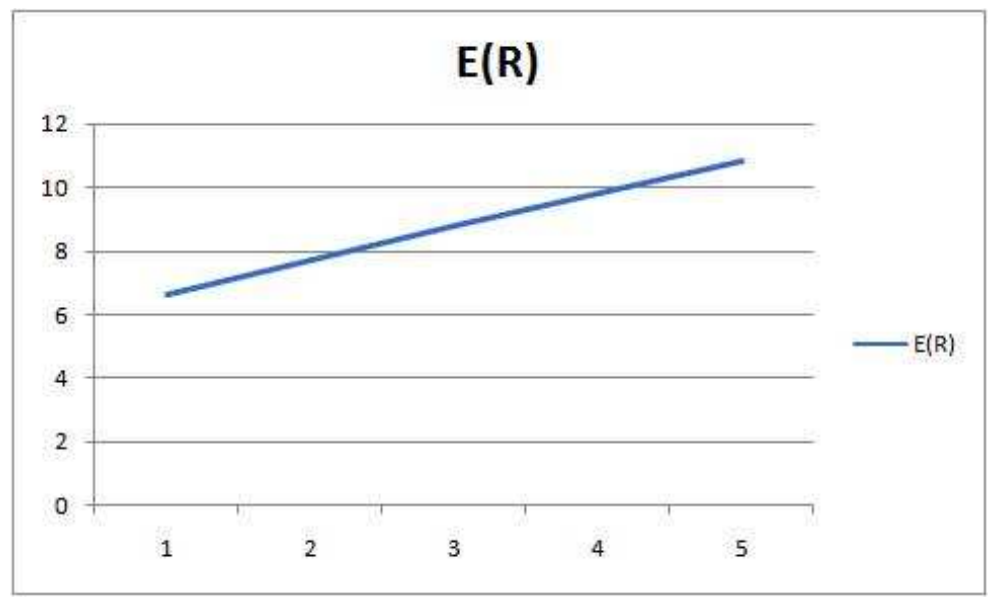

We may note that when $c$ increases (expected time to season decreases) $E(T)$ decreases and $E(R)$ increases.

\section{Acknowledgments}

The authors thank the management of VelTech MultiTech Dr. Rangarajan Dr. Sakuntala Engineering College, and Veltech DR. RR and DR. SR. Technical University, Avadi, Chennai for providing necessary facilities to bring out this research paper in a very short time.

\section{References}

[1] K. Arrow, S. Karlin, H. Scarf, Studies in the Mathematical Theory of Inventory and Production, Standard University Press, Standard, California (1958).

[2] J.K. Daniel, R. Ramanarayanan, An $(S, s)$ inventory system with rest periods to the server, Naval Research Logistics, 35 (1988), 119-123.

[3] Kun-Shan Wu, Ouyang Liang-Yuh, $(Q, r, L)$ inventory model with defective items, Computer and Industrial Engineering, 39 (2001), 173-185.

[4] S. Murthy and R. Ramanarayanan, Two ordering levels inventory system with different lead times and rest time to the server, Inter. J. of Applied Math., 21, No. 2 (2008), 265-280. 
[5] S. Murthy and R. Ramanarayanan, Two $(s, S)$ inventories with perishable units, The Journal of Modern Mathematics and Satistics, 2, No. 3 (2008), 102-108.

[6] S. Murthy and R. Ramanarayanan, Inventory system exposed to Calamity with SCBZ arrival property, The Journal of Modern Mathematics and Satistics, 2, No. 3 (2008), 109-119.

[7] S. Murthy and R. Ramanarayanan, General analysis of $(s, S)$ inventory system with defective supplies, Inter. J. of Applied Math., 21, No. 3 (2008), 495-507.

[8] R. Ramanarayanan and M.J. Jacob, General analysis of $(S, s)$ inventory systems with random lead time and bulk demand, Cashieru de C.E.R.O., No-s: 3-4 (1988), 119-123. 